\title{
The Flare Detection in the Two Color Crossed Array Detectors Infrared Seeker
}

\author{
S. Y. AlchekhYasin \\ Department of Electrical Eng \\ IUST University \\ Islamic Republic of Iran
}

\author{
A. R.Erfanian \\ Department of Electrical Eng \\ Maleke-Ashtar University \\ Islamic Republic of Iran \\ A. Mohammadi \\ Department of Aerospace Eng \\ Maleke-Ashtar University \\ Islamic Republic of Iran
}

\author{
M. R. Mosavi \\ Department of Electrical Eng \\ IUST University \\ Islamic Republic of Iran
}

\begin{abstract}
One of the important parts of the infrared seeker is the counter countermeasure part. Seeking in a field of view with the existence of jammers is not simple and needs effective algorithms. In this paper, several methods of the detection in a crossed array trachers (CAT) seeker will be designed, simulated and evaluated. These methods depend on detecting

the appearance of the flare using analzing the two channels signals.
\end{abstract}

\section{Keywords}

Infrared Seeker, Counter Countermeasure, Flare Detection, Two-Color Algorithm, Crossed Array Detectors (CAT), Field of view (FOV).

\section{INTRODUCTION}

The IR counter-countermeasure (IRCCM) built into advanced missiles have two parts. The first part is detecting the existence or the appearance of the flares in the seeker field of view (FOV) and activating the process for overcoming these jammers; sometime this part is called switch part. The second part is applying the IRCCM algorithms to overcome the jammers effect on the seeking process; sometime this part is called response part [1-2].

A missile must detect the flare before initiating a counter. When a flare is detected by a seeker in an advanced IR missile, the seeker will switch on the IRCCM response to reject the flare . Both the switch and response must operate properly to successfully reject the flare and continue tracking the target. There are many different switch and response techniques available to the missile designers, thus a device that is capable of decoying one advanced IR missile type may be totally ineffective against another advanced IR missile.

Detecting the existence or the appearance of the flares in a short time is a critical task, as increasing the required time of detection gives the flare the required time to push the real target out of the effective work region of the FOV, and as a result missing the real target [2-5].

In this paper, it is stated several methods for detecting the flares in the FOV of a crossed array detectors seeker (CAT) or a four crossed slits reticle seeker. Firstly, detecting using the amplitude of one of the two channels in the two color seeker will be stated. Secondly, detecting the flares using the ratio factor between the two channels in several ways will be discussed.
All the modeling and simulation tasks and processes will be designed and developed using the MATLAB ${ }^{\circledR}$ tools and packages.

The structure of the crossed ship reticle or the crossed four slits reticle (Actually it is equivalent to a reticle with four crossed array detectors CAT), which is a stationary reticle type, will be stated with the design parameters. This type employs a fixed reticle, with radius $\mathrm{Ra}$ and $\mathrm{N}=4$ transparent sectors or slits, and a slightly tilted rotating mirror or lens (with spinning frequency fm ) to sweep the Target Image Spot (TIS) along a circular path on the reticle Target Imaging Circle (TIC) with constant radius (RN), as shown in Fig. 1,2. The distance of the non-concentric TIC centre and its phase relatively to the reticle centre define the position of the target in the FOV [6-10].

In Fig 2 the defined parameters:

- D: the spoke width or the detector width (pixel).

- $\quad$ L: the spoke length or the detector length (pixel).

- $\quad$ Ra: the reticle radius (FOV radius) (pixel).

- $\mathrm{R}_{\mathrm{N}}$ : the target imaging circle (TIC) (pixel).

In this study, theses parameters take the following values: ( $D=70, L=850, R N=615, R a=885$ ) unit or pixel. When the TIC is not concentrate with the reticle, which means that the target position is not on the centre of the FOV, the imaging circle centre (or the target position) parameters $(\mathrm{aT}(\mathrm{t}), \beta 0(\mathrm{t}))$ can be defined.

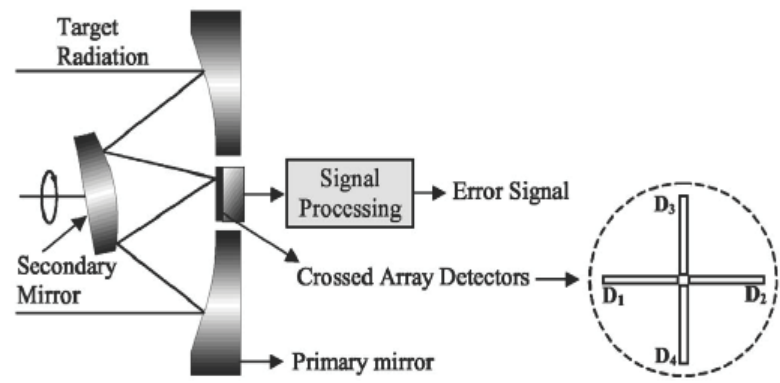

Fig 1: The cross ship reticle (equivalent to a reticle with four crossed array detectors). 


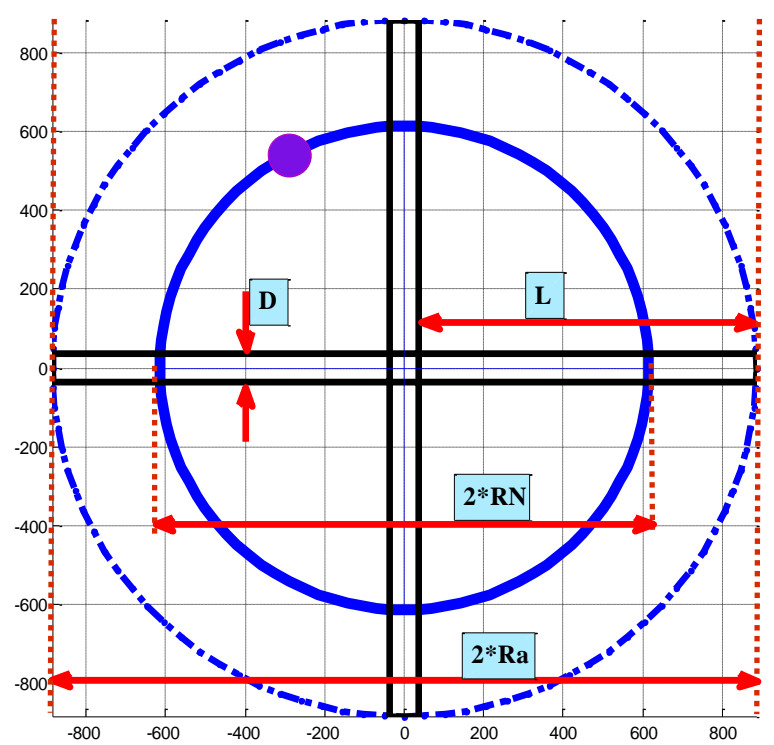

Fig. 2 The design parameters of a CAT reticle (D,L, Ra, RN).

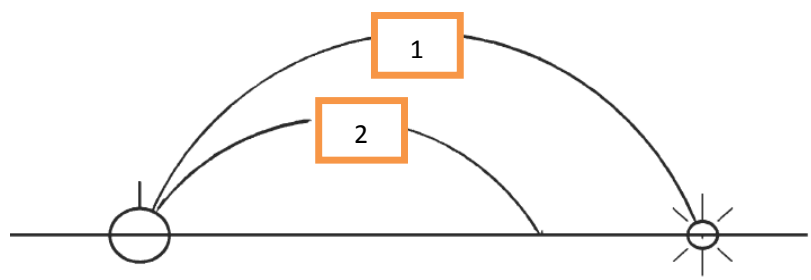

Fig 3: The movement profile of the flare in the space.

The used reticle model takes the reticle parameters (D, L, $\mathrm{Ra}, \mathrm{RN}$ ) as inputs and gives the output signal up to the target position parameters, such the coordination in the Field Of View (FOV). For modelling the two-color seeker, a model of a detector with variant band limits, $[\lambda \min 1, \lambda \max 1]$ for the $(\mathrm{M}$ band) in mid IR region and $[\lambda \min 2, \lambda \max 2]$ for the $(\mathrm{N}$ band) in near IR region. The detectors outputs (M1T,M2T,M1J,M2J) will forms the two color channels by combining with the reticle outputs [11-13]. An as result, six signals will be produced using the final used model, as shown in Fig 4:

- Y1(ch1) and Y2(ch2) are the main channel and the secondary channel signals.

- Yt1 and Yt2 are the signals resulting from the existence of the real target in the FOV in the two channels respectively.

- $\mathrm{M}_{\mathrm{n}-\mathrm{kNm}}$ : the number of the pulses exist in this spinning period ]n-k*Nm, n-k*Nm+Nm], or $\mathrm{k}$-th previous spinning period.

- $\mathrm{B}_{\mathrm{n}-\mathrm{kNm}}[\mathrm{i}]$ : the maximum of the $\mathrm{i}-\mathrm{th}$ pulse in this period, so $1 \leq \mathrm{i} \leq \mathrm{M}_{\mathrm{n}-\mathrm{kNm}}$.

- $\mathrm{A}_{\mathrm{n}}[\mathrm{k}]=\max \left\{\mathrm{B}_{\mathrm{n}-\mathrm{kNm}}[\mathrm{i}]: 1 \leq \mathrm{i} \leq \mathrm{M}_{\mathrm{n}-\mathrm{kNm}}\right\}=\max \{\mathrm{x}[\mathrm{j}]: \mathrm{n}-$ $k \cdot N m \leq j \leq n-k \cdot N m+N m\}$ the max value on the spinning period $] \mathrm{n}-\mathrm{k} * \mathrm{Nm}, \mathrm{n}-\mathrm{k} * \mathrm{Nm}+\mathrm{Nm}]$.

For simplifying, as shown in Fig 6:

- $\mathrm{M}_{\mathrm{k}}=\mathrm{M}_{\mathrm{n}-\mathrm{kNm}}, \mathrm{B}_{\mathrm{k}}[\mathrm{i}]=\mathrm{B}_{\mathrm{n}-\mathrm{kNm}}[\mathrm{i}]$.
- $Y j 1$ and $Y j 2$ are the signals resulting from the existence of a flare in the FOV in the two channels respectively.

The flare is modeled by designing a flare temperature profile generator and a flare position generator. The flare temperature profile determines the profile of the flare signal amplitude, so the profile of the main channel amplitude with time. The output temperature is given by the following equations:

$$
T_{F}=T_{\text {max }} *\left\{\begin{array}{lr}
0 & \left(t<t_{0}\right) \text { or }\left(T_{f}<t\right) \\
1-e^{-\frac{t-t_{0}}{T_{r}}} & t_{0} \leq t<t_{0}+T_{1} \\
e^{-\frac{\left(t-T_{r}-t_{0}\right)}{T_{b}}} & t_{0}+T_{1} \leq t \leq t_{0}+T_{f}
\end{array}\right.
$$

where $\mathrm{t} 0$ is the launch instant, $\mathrm{Tf}$ is the flare life duration, $\mathrm{Tr}$ is the rise time constant, $\mathrm{T} 1$ is the rise duration, $\mathrm{Tb}$ is the burn time constant, and Tmax is the maximum temperature. This temperature TF variable will be the flare temperature input of the seeker model [14].

The flare movement generator, as shown in Fig 3, determines the flare position in the FOV with time, the main parameters of that are the movement of the flare in the space and the distance from the missile to the flare [15-16]. Taking into account the FOV angle is usually \pm 1 degee, the FOV space will be a disc with a radius of $\left(\mathrm{R}^{*} 17\right) \mathrm{m}$ where $\mathrm{R}(\mathrm{km})$ is the distance from the missile and the flare. In addition to that, the initial speed or the launch speed of the flare is $[20-30] \mathrm{m} / \mathrm{s}$. As result of that, the flare movement scenario in the FOV can be determined. Up to the practices previous ranges, and taking into accounts that reticle radius is ( 885 pixls) the speed of the flares in the FOV in the simulation in the range [500,2500] pixel/s, which means that the flare remains in the FOV $[0.35,1.5] \mathrm{s}$

\section{FLARE DETECTION USING THE RATE OF THE AMPLITUDE INCREASING IN THE MAIN CHANNEL.}

For explaining this method, several parameters have to be defined. On the period ]t-Tm,t], or digitally on ]n-Nm,n], as shown in Fig 5:

- $\mathrm{Tm}=\mathrm{Nm} * \mathrm{Ts}: \mathrm{Ts}$ is the sampling period.

- $\mathrm{M}_{\mathrm{n}-\mathrm{Nm}}$ : the number of the pulses exist in this spinning period ]n-Nm,n].

- $\quad \mathrm{B}_{\mathrm{n}-\mathrm{Nm}}[\mathrm{i}]$ : the maximum of the $\mathrm{i}$-th pulse in this period, so $1 \leq \mathrm{i} \leq \mathrm{M}_{\mathrm{n}-\mathrm{Nm}}$

- $\quad \mathrm{A}_{\mathrm{n}}[1]=\max \left\{\mathrm{B}_{\mathrm{n}-\mathrm{Nm}}[\mathrm{i}]: 1 \leq \mathrm{i} \leq \mathrm{M}_{\mathrm{n}-\mathrm{Nm}}\right\}=\max \{\mathrm{x}[\mathrm{j}]: \mathrm{n}-$ $\mathrm{Nm} \leq \mathrm{j} \leq \mathrm{n}\}$ the max value on the spinning period $] \mathrm{n}$ $\mathrm{Nm}, \mathrm{n}]$.

So it can be generalized on any previous spinning period, or digitally on ]n-k*Nm, n-k*Nm+Nm]

- $\mathrm{A}[\mathrm{k}]=\mathrm{A}_{\mathrm{n}}[\mathrm{k}]$

- $\{\mathrm{A}[\mathrm{k}]: 1 \leq \mathrm{k} \leq \mathrm{N}\}$ : the maximum of the $\mathrm{N}$ previous spinning period.

So the condition of detecting the flare is:

$A[1]>$ maxthrd $* A[1+$ maxdelay where:

- maxthrd : is the threshold coefficient.

- maxdelay: is the delay coefficient

The parameters maxthrd and maxdelay will be studied in the following task. Actually, in this method of detection, the first 
note is that the flare movement profile and the time burn will not affect in the flare detection. This because these parameters are not important in the detection process while the flare still in the FOV. On the contrast, they are very important in the IRCCM algorithms. As result, the study will focus on the effect of the rise time of the flare temperature profile in the detection using this method and choosing the optimal values of the parameters maxthrd and maxdelay.

In the scenario the target will be in the centre and the flare moves in an angle 45degree outing the reticle from the target with a suitable speed ( the flare stay on the reticle about $0.5 \mathrm{~s}$ ). The loop is open to avoid tracking the flare.

In the following, the parameters of the flare temperature profile will be changed and the values of (maxthrd, maxdelay) will be gotten for every case. As a result, the optimal values of (maxthrd, maxdelay) will appear .As shown in the Fig 7, the flare temperature profile parameters effect on the profile of the main channel signal and as result it's A[1] which represents the coverage signal of it, as shown in Fig 7 (b). It is clear that, the parameter maxdelay has to be as small as possible for detecting the flare as early as possible. But it cannot be so small, as the duration between the separation of the flare from the target and its starting burning instant takes minimum time not less than $50 \mathrm{~ms}$. On the other hand, clearly it cannot so big. As result, three values are chosen to be studied maxdelay $=\{5,10,15\}$.

Also, the parameter maxthrd has to be as small as possible for detecting the flare as early as possible. But it cannot be so small to avoid false alarm, as the main channel signal fluctuates with a rate that increases with existence of important noise or hot background. On the other hand, clearly it cannot so big to avoid miss detecting. As result, maxthrd has to be chosen more than 1.2 and as minimum as possible. For this purpose, $\mathrm{A}[1,5,10,15]$ will be drawn, then $\mathrm{A}[1] / \mathrm{A}[5]$, $\mathrm{A}[1] / \mathrm{A}[10]$ and $\mathrm{A}[1] / \mathrm{A}[15]$ will be calculated to check the suitable values for (maxthrd, maxdelay), as show in Fig 7(d).

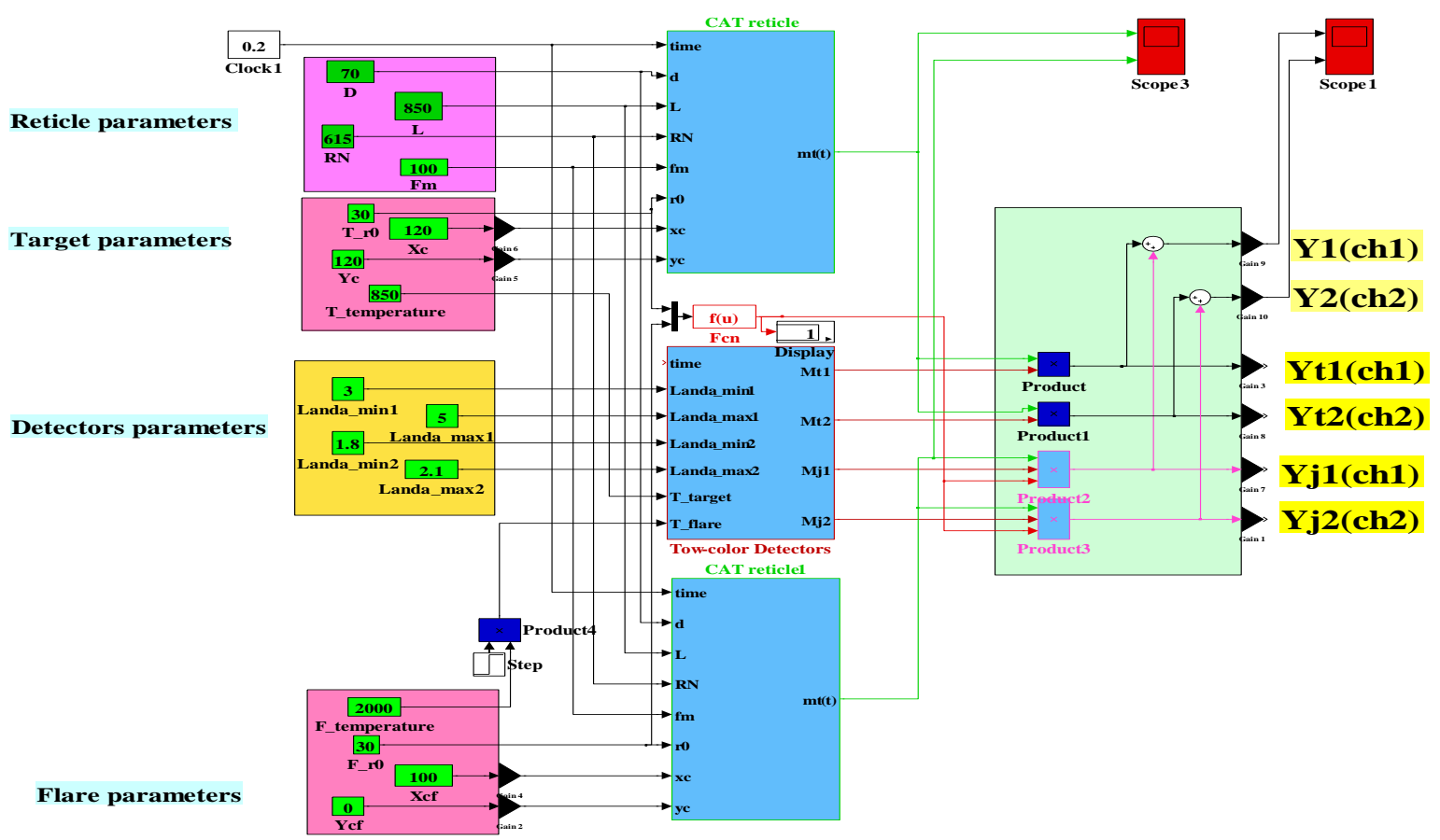

Fig 4: The final model with the inputs (reticle parameters, detectors parameters, target parameters, and flare parameters)and the outputs $($ Y1(ch1), Y2(ch2), Ref=Y1T, Y2T, Y1F , Y2F ).

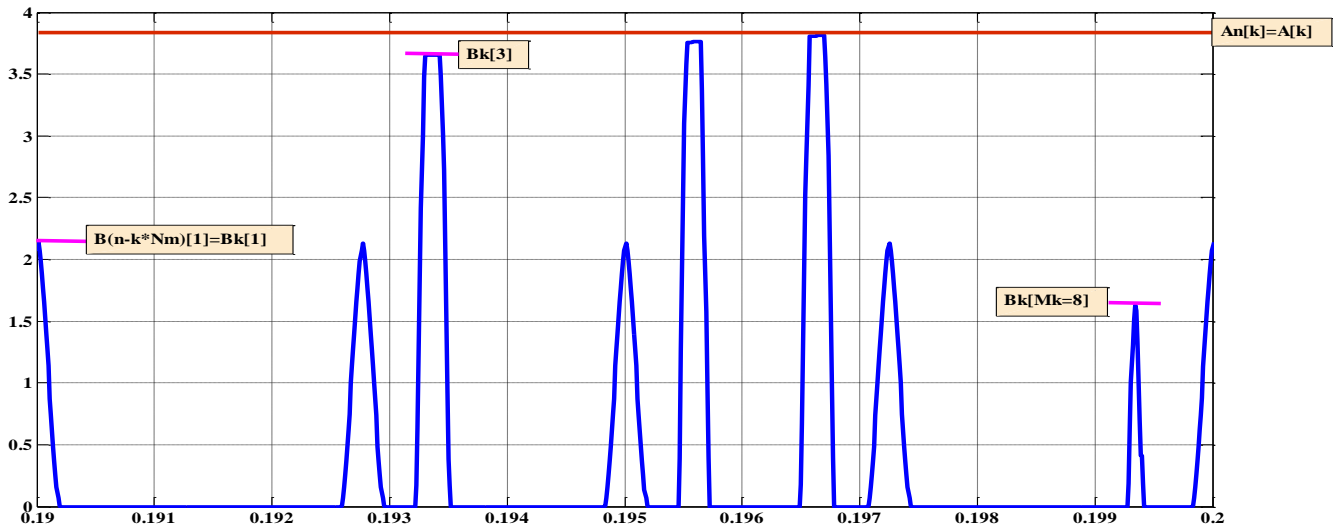

Fig 5: The parameters on one spinning period. 


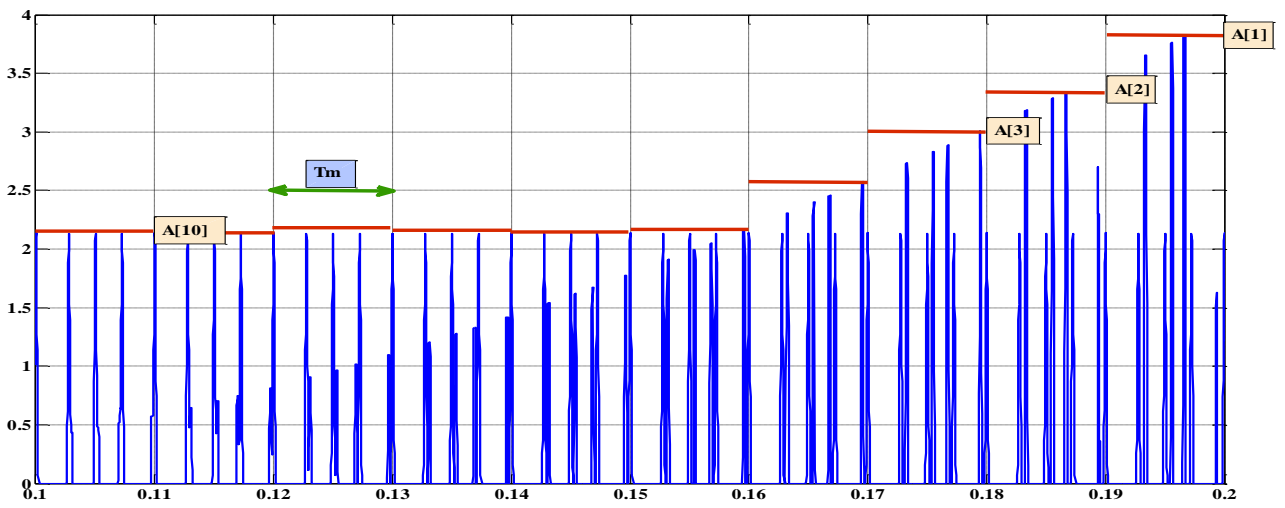

Fig 6: The parameters on several previous spinning periods.

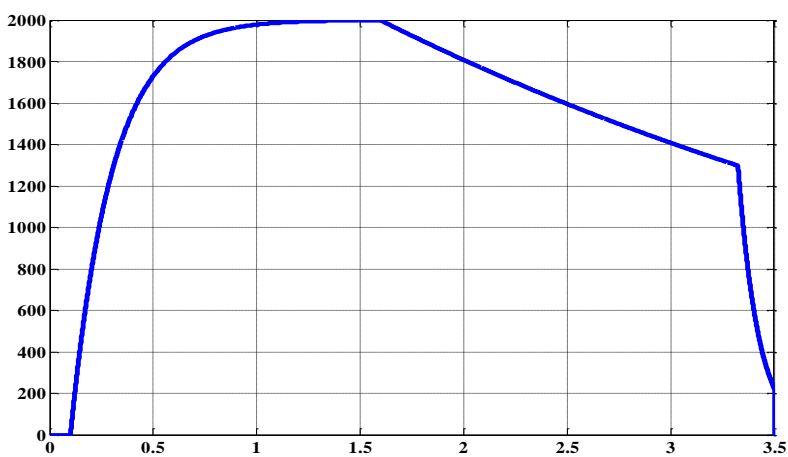

(a)

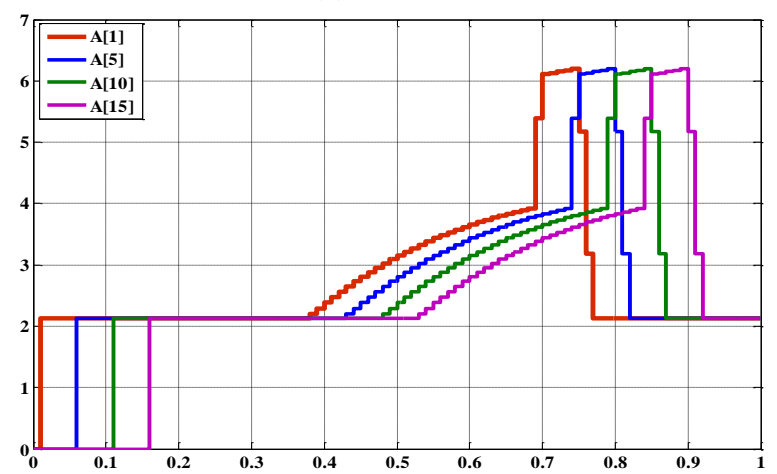

(c)

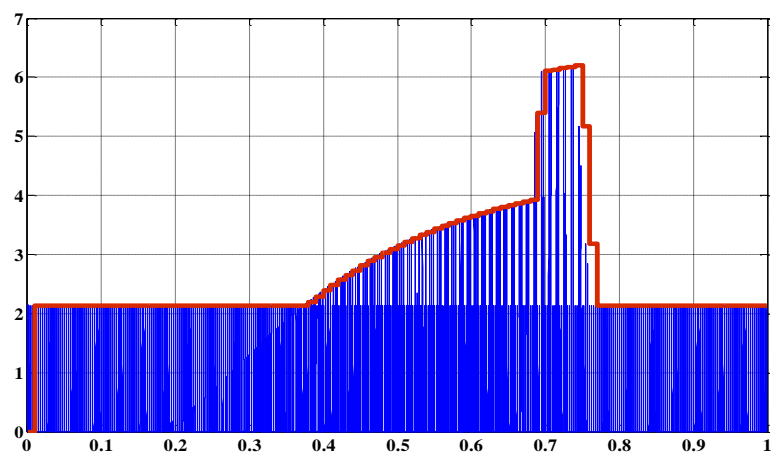

(b)

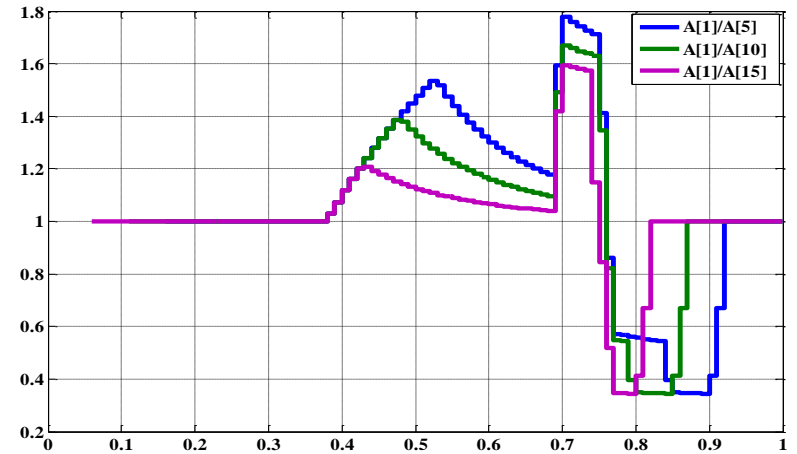

(d)

Fig 7: The process for finding (maxthrd, maxdelay), in this case: $\operatorname{Tr}=0.2$, $T r i s e=1.5 \mathrm{~s}, \operatorname{Tmax}=2000 \mathrm{k}, \mathrm{Tf}=3.5 \mathrm{~s}, \mathrm{~Tb}=4$

(a) flare temperature profile (b) main channel amplitude profile (c) $\mathrm{A}[\mathbf{1 , 5 , 1 0 , 1 5 ]}$ (d) $\mathrm{A}[\mathbf{1}] / \mathrm{A}[\mathbf{5 , 1 0 , 1 5 ]}$.

In Fig 7 , the data is gotten for ( $\operatorname{Tr}=0.2$, Trise $=1.5 \mathrm{~s}$, $\mathrm{Tmax}=2000 \mathrm{k}, \mathrm{Tf}=3.5 \mathrm{~s}, \mathrm{~Tb}=4)$. And in Fig 8 (a), the temperature profiles are draw for same parameters except Trise $=\{0.5,1.0,1.5\} \mathrm{s}$. It is clear, in this figure, that the three cases share the values on the interval $[0,0.6] \mathrm{s}$ which means that they share the main channel signal in this interval. Taking into account that this time interval is the interval where the flare can be detected. And as result, it is enough to study only one value of Trise which is the minimum $0.5 \mathrm{~s}$, as shown in Fig 8(b).

The results of this approach for several values of $\operatorname{Tr}$ can be resumed in the following tables $(1,2,3)$. In the first table the suitable ranges of the parameter maxthrd for several cases of $\mathrm{Tr}$ are stated. In the second table the required time for the accepted cases in table 1 are stated. The third table show the validity duration time for these cases.
Table 1. The suitable ranges of maxthrd

\begin{tabular}{|c|c|c|c|c|}
\hline $\begin{array}{c}\text { Maxdelay } \\
(\mathbf{T m})\end{array}$ & $\mathbf{T r}=\mathbf{0 . 1 0}$ & $\mathbf{T r}=\mathbf{0 . 1 5}$ & $\mathbf{T r}=\mathbf{0 . 2 0}$ & $\mathbf{T r}=\mathbf{0 . 3 0}$ \\
\hline 5 & {$[1.2-1.4]$} & {$[1.2-1.25]$} & {$[1.2-1.2[$} & {$[1.2-1.15[$} \\
\hline 10 & {$[1.2-1.6]$} & {$[1.2-1.45]$} & {$[1.2-1.4]$} & {$[1.2-1.3]$} \\
\hline 15 & {$[1.2-1.8]$} & {$[1.2-1.60]$} & {$[1.2-1.5]$} & {$[1.2-1.4]$} \\
\hline
\end{tabular}

Table 2. The required time for detecting the flare for (maxthrd=1.3)

\begin{tabular}{|c|c|c|c|c|}
\hline $\begin{array}{c}\text { Maxdelay } \\
(\mathbf{T m})\end{array}$ & $\mathbf{T r}=\mathbf{0 . 1 0}$ & $\mathbf{T r}=\mathbf{0 . 1 5}$ & $\mathbf{T r}=\mathbf{0 . 2 0}$ & $\mathbf{T r}=\mathbf{0 . 3 0}$ \\
\hline 10 & $0.18 \mathrm{~s}$ & $0.25 \mathrm{~s}$ & $0.35 \mathrm{~s}$ & $0.50 \mathrm{~s}$ \\
\hline 15 & $0.18 \mathrm{~s}$ & $0.25 \mathrm{~s}$ & $0.35 \mathrm{~s}$ & $0.52 \mathrm{~s}$ \\
\hline
\end{tabular}


Table 3.The validity duration for detecting the dlare for (maxdelay=15).

\begin{tabular}{|c|c|c|c|c|}
\hline Maxthrd & Tr=0.10 & Tr=0.15 & Tr=0.20 & Tr=0.30 \\
\hline 1.3 & $0.17 \mathrm{~s}$ & $0.17 \mathrm{~s}$ & $0.15 \mathrm{~s}$ & $0.05 \mathrm{~s}$ \\
\hline 1.4 & $0.14 \mathrm{~s}$ & $0.13 \mathrm{~s}$ & $0.09 \mathrm{~s}$ & $0.01 \mathrm{~s}$ \\
\hline
\end{tabular}

From these tables:

- $\quad($ maxdelay=5) is not acceptable (table 1$)$.

- $\quad($ maxdelay $\geq 10)($ table 1$)$.

- (maxthd=1.3) is the suitable choose .

- $\quad(\operatorname{maxthrd}=1.3$, maxdelay $=10)$ or $\quad(\operatorname{maxthrd}=1.3$, maxdelay=15) are suitable choose (table 1).

- If the continuity of the condition is taken into account the (maxthrd=1.3 , maxdelay=15) is the good choose. The continuity is used to assure the alarm. The continuity of (maxthrd=1.3) for $0.05 \mathrm{~s}$ is better than (maxthrd=1.4), as shown in table 3 .

The final result, the more suitable values are : (maxthrd=1.3, maxdelay $=15$ ). The flowchart of applying this method with the chosen values for the parameters is chosen in Fig 9.

\section{DETECTION USING THE RATE OF THE AMPLITUDE INCREASING IN THE SECONDARY CHANNEL.}

Using the same approach used in the previuos part, the detection using the amplitude increasing rate in the secondary channel is studied. Actually, the parameters are calculated by the same manner, the optimum values are (maxthrd2=3.0, maxdelay2 $=15$ ). As it is expected maxthrd in the secondary channel has larger value than that of the main channel; this results from the fact that the secondary channel amplitude increases with higher rate when the flare appears.

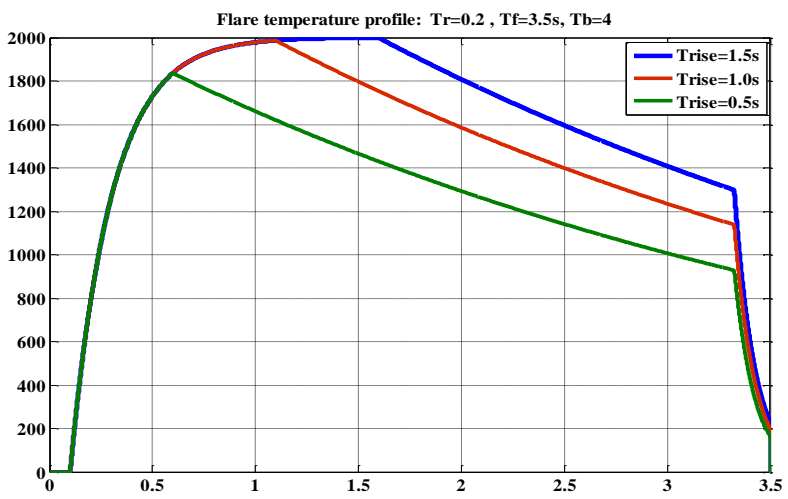

(a)

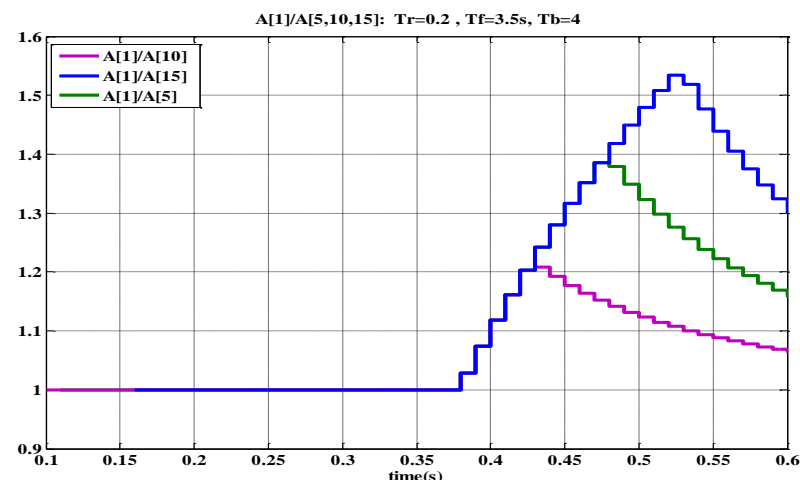

(b)

Fig 8: In this case $(T r=0.2, T \max =2000 \mathrm{k}, T f=3.5 \mathrm{~s}, \mathrm{~Tb}=4)$ and Trise $=\{0.5,1.0,1.5\} \mathrm{s}:$ (a) flare temperature profile (b) $\mathrm{A}[\mathbf{1}] / \mathrm{A}[\mathbf{5 , 1 0 , 1 5 ]}$.

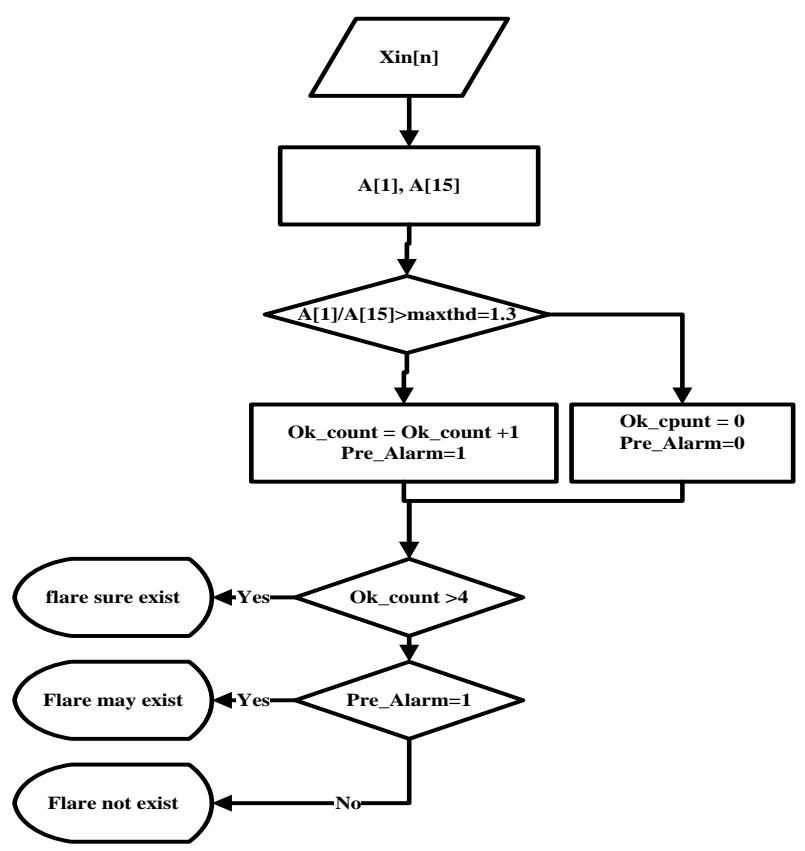

Fig 9: The flowchart of the detection using the amplitude increasing rate in the main channel in the CAT seeker 


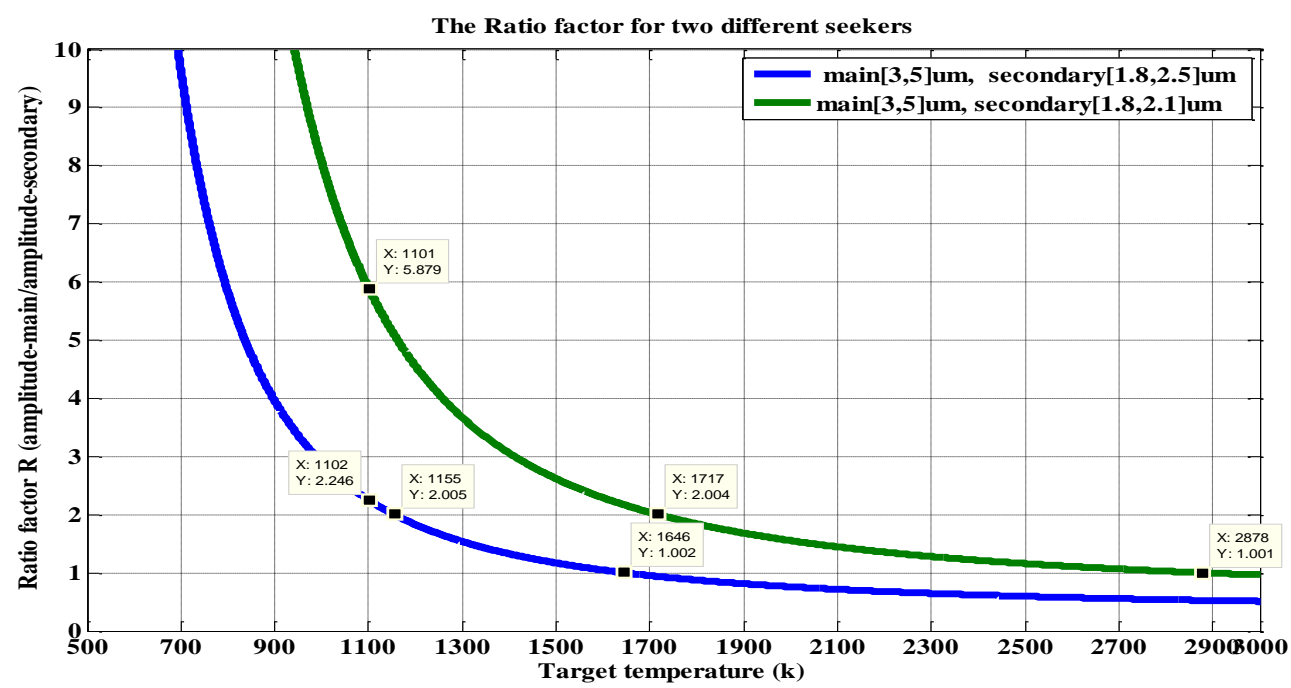

Fig 10: The Ratio Factor (R) of the two-color seekers.

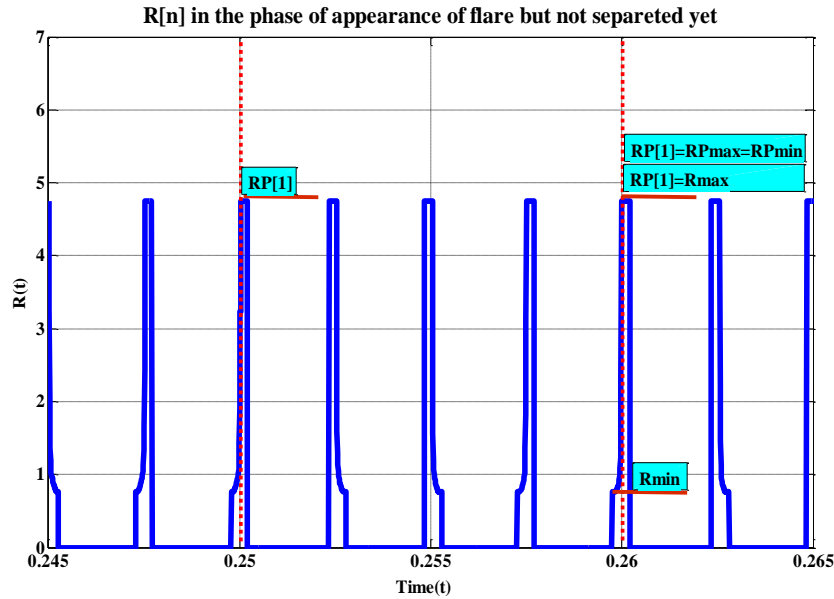

(a)

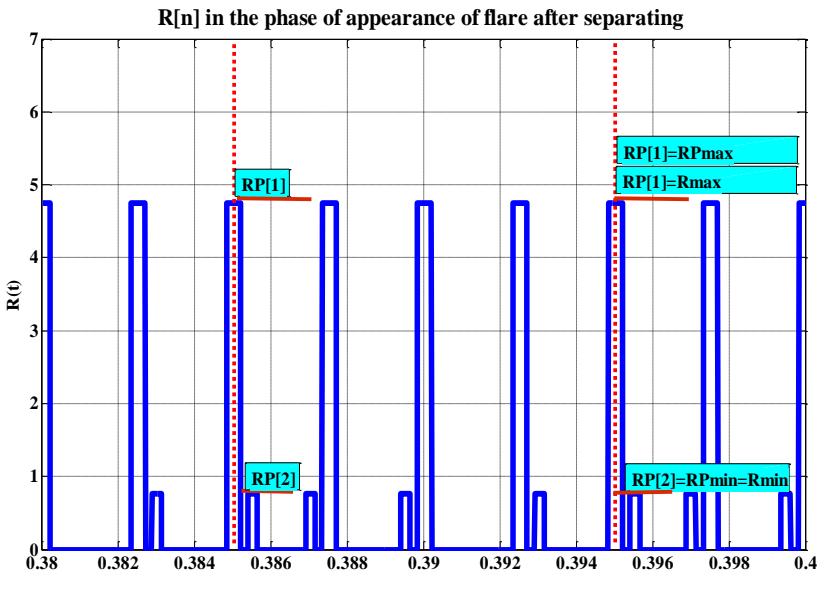

(b)

Fig 11: Ratio Factor R (Rmax, Rmin, dR) and Ratio Pulse Factor RP (RPmax, RPmin, dRP) definition in the main channel.

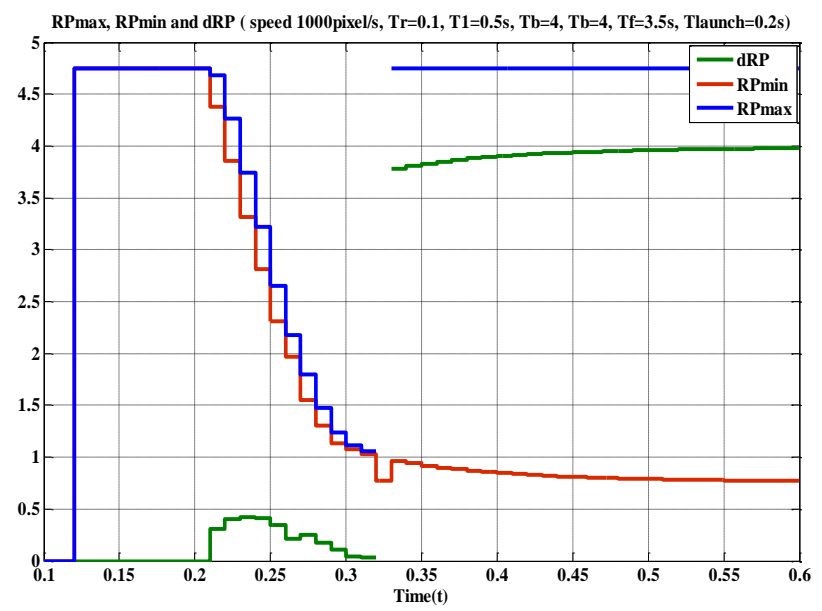

(a)

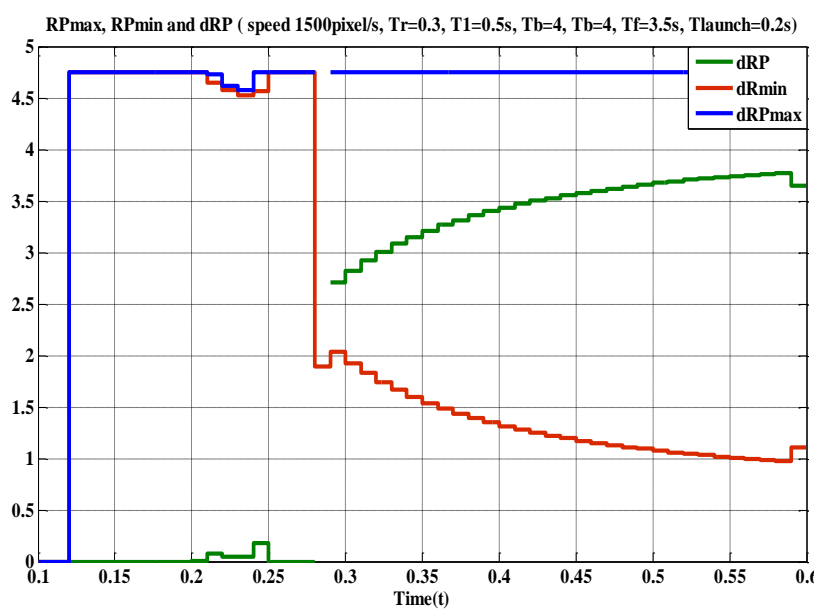

(b)

Fig 12 Ratio Factor $R(R \max , R \min , d R)$ in the main channel for a Flare $(T$ lanuch $=0.2 \mathrm{~s}, \mathrm{Trise}=0.5 \mathrm{~s}, \mathrm{ff}=3.5 \mathrm{~s}, \mathrm{~Tb}=4$, $\operatorname{Tmax}=2000 \mathrm{k}, \mathrm{Tmin}=850 \mathrm{k}$ ): $\quad$ (a) $\mathrm{Tr}=0.1$, speed $=1000 \mathrm{pixel} / \mathrm{s} \quad$ (b) $\mathrm{Tr}=0.3$, , speed $=1500 \mathrm{pixel} / \mathrm{s}$ 


\section{DETECTION USING THE RATIO THE AMPLITUDES OF THE TWO CHANNELS.}

For detecting using the ratio factor $(\mathrm{R})$ between the two channels it is important to get the value of this factor for all the target temperature cases. The relative response of the two channels depends to the two bands $[\lambda \min 1, \lambda \max 1]$ and $[\lambda \min 2, \lambda \max 2]$. This characteristic is resulted from the integrated nature of the detectors. In Fig 10 the values of this factor in two cases for two-color seekers, in the both seekers. The main channel band is $[\lambda \min 1=3 \mu \mathrm{m}, \lambda \max 1=5 \mu \mathrm{m}]$, while the secondary channel in the first is $[\lambda \min 2=1.8 \mu \mathrm{m}$, $\lambda \max 2=2.5 \mu \mathrm{m}]$ and $[\lambda \min 2=1.8 \mu \mathrm{m}, \lambda \max 2=2.1 \mu \mathrm{m}]$ in the second one. In the following study the first seeker will be considered. Important values are inserted in the table 4.

Table 4.Important values in the response of the two-color seeker

\begin{tabular}{|c|c|}
\hline Ratio Factor & T(k) \\
\hline $\mathrm{R}=1$ & 1646 \\
\hline $\mathrm{R}=2$ & 1155 \\
\hline $\mathrm{R}=2.25$ & 1100 \\
\hline
\end{tabular}

For explaining this method, several parameters have to be defined. On the period ]t-Tm,t], or digitally on ]n-Nm,n]:

- $\mathrm{M}_{\mathrm{n}-\mathrm{Nm}}$ :the pulses number in this spinning period ]n$\mathrm{Nm}, \mathrm{n}]$.

- $\mathrm{R}[\mathrm{n}]=x \operatorname{main}[\mathrm{n}] / \mathrm{xsec}[\mathrm{n}]=\mathrm{xp}[\mathrm{n}] / \mathrm{xp} 2[\mathrm{n}]$ the ratio at the instant $\mathrm{n}$.

- $\operatorname{Rmax}_{n-N m}=\max \{R[n]: n$ in $\left.\left.] n-N m, n\right]\right\}$ the max ratio in the spinning period ]n-Nm,n].

- $\operatorname{Rmin}_{\mathrm{n}-\mathrm{Nm}}=\min \{\mathrm{R}[\mathrm{n}]: \mathrm{n}$ in $\left.\left.] \mathrm{n}-\mathrm{Nm}, \mathrm{n}\right]\right\}$ the $\min$ ratio in the spinning period $] \mathrm{n}-\mathrm{Nm}, \mathrm{n}]$.

- $\quad \mathrm{RP}_{\mathrm{n}-\mathrm{Nm}}[\mathrm{i}]$ : the Ratio at the maximum of the $\mathrm{i}$-th pulse in this period, so $1 \leq \mathrm{i} \leq \mathrm{M}_{\mathrm{n}-\mathrm{Nm}}$.

- $\operatorname{RPmax}_{\mathrm{n}-\mathrm{Nm}}=\max \left\{\mathrm{RP}_{\mathrm{n}-\mathrm{Nm}}[\mathrm{i}]: 1 \leq \mathrm{i} \leq \mathrm{M}_{\mathrm{n}-\mathrm{Nm}}\right\}$ the $\max$ ratio of pulses in the spinning period $] \mathrm{n}-\mathrm{Nm}, \mathrm{n}]$.

- $\quad R P \min _{n-N m}=\min \left\{\mathrm{RP}_{\mathrm{n}-\mathrm{Nm}}[\mathrm{i}]: 1 \leq \mathrm{i} \leq \mathrm{M}_{\mathrm{n}-\mathrm{Nm}}\right\}$ the min ratio of pulses in the spinning period ]n-Nm,n].

So it can be generalized on any previous spinning period, or digitally on ]n-k*Nm, $\mathbf{n}-\mathbf{k} * \mathbf{N m}+\mathbf{N m}]$ :

- $\mathrm{M}_{\mathrm{n}-\mathrm{kNm}}$ : the pulses number in this spinning period ]n$\mathrm{k} * \mathrm{Nm}, \mathrm{n}-\mathrm{k} * \mathrm{Nm}+\mathrm{Nm}]$, or $\mathrm{k}$-th previous spinning period.

- $R \max { }_{n-k * N m}=\max \{R[n]: n$ in $\left.\left.] n-k * N m, n-k * N m+N m\right]\right\}$ the max ratio in the spinning period $] \mathrm{n}-\mathrm{k}^{*} \mathrm{Nm}$, n$\mathrm{k} * \mathrm{Nm}+\mathrm{Nm}]$.

- $\quad \operatorname{Rmin}{ }_{\mathrm{n}-\mathrm{k} * \mathrm{Nm}}=\min \{\mathrm{R}[\mathrm{n}]: \mathrm{n}$ in $\left.\left.] \mathrm{n}-\mathrm{k} * \mathrm{Nm}, \mathrm{n}-\mathrm{k} * \mathrm{Nm}+\mathrm{Nm}\right]\right\}$ the min ratio in the spinning period $] \mathrm{n}-\mathrm{k} * \mathrm{Nm}$, n$\left.\mathrm{k}^{*} \mathrm{Nm}+\mathrm{Nm}\right]$.

- $\quad \mathrm{RP}_{\mathrm{n}-\mathrm{k} * \mathrm{Nm}}[\mathrm{i}]:$ the Ratio at the maximum of the $\mathrm{i}$-th pulse in this period, so $1 \leq \mathrm{i} \leq \mathrm{M}_{\mathrm{n}-\mathrm{k}^{*} \mathrm{Nm}}$.

- $\operatorname{RPmax}_{\mathrm{n}-\mathrm{k}^{*} \mathrm{Nm}}=\max \left\{\mathrm{RP}_{\mathrm{n}-\mathrm{k}^{*} \mathrm{Nm}}[\mathrm{i}]: 1 \leq \mathrm{i} \leq \mathrm{M}_{\mathrm{n}-\mathrm{k}^{*} \mathrm{Nm}}\right\}$ the max ratio of pulses in the spinning period ]n-k*Nm, n$\left.\mathrm{k}^{*} \mathrm{Nm}+\mathrm{Nm}\right]$
- $\quad \operatorname{RPmin}_{\mathrm{n}-\mathrm{k}^{*} \mathrm{Nm}}=\min \left\{\mathrm{RP}_{\mathrm{n}-\mathrm{k}^{*} \mathrm{Nm}}[\mathrm{i}]: 1 \leq \mathrm{i} \leq \mathrm{M}_{\mathrm{n}-\mathrm{k}^{*} \mathrm{Nm}}\right\}$ the min ratio of pulses in the spinning period $] n-k * N m, n-$ $\mathrm{k} * \mathrm{Nm}+\mathrm{Nm}]$.

For simplifying, as shown in Fig 11.:

- $\mathrm{M}_{\mathrm{k}}=\mathrm{M}_{\mathrm{n}-\mathrm{kNm}}, \mathrm{RP}_{\mathrm{k}}[\mathrm{i}]=\mathrm{RP}_{\mathrm{n}-\mathrm{k} * \mathrm{Nm}}[\mathrm{i}]$.

- $R \max [\mathrm{k}]=\operatorname{Rmax}_{\mathrm{n}-\mathrm{k} * \mathrm{Nm}} \cdot\{\operatorname{Rmax}[\mathrm{k}]: 1 \leq \mathrm{k} \leq \mathrm{N}\}$ are the Rmax of the $\mathrm{N}$ previous spinning period.

- $R \min [\mathrm{k}]=\operatorname{Rmix}_{\mathrm{n}-\mathrm{k}^{*} \mathrm{Nm}} \cdot\{\operatorname{Rmin}[\mathrm{k}]: 1 \leq \mathrm{k} \leq \mathrm{N}\}$ are the Rmin of the $\mathrm{N}$ previous spinning period.

- $\quad \operatorname{RPmax}[\mathrm{k}]=\operatorname{RPmax}_{\mathrm{n}-\mathrm{k} * \mathrm{Nm}} \cdot\{\operatorname{RPmax}[\mathrm{k}]: 1 \leq \mathrm{k} \leq \mathrm{N}\}$ are the RPmax of the $\mathrm{N}$ previous spinning period.

- $R P \min [\mathrm{k}]=\mathrm{RPmin} \operatorname{m-k}^{*} \mathrm{Nm} \cdot\{\operatorname{RPmin}[\mathrm{k}]: 1 \leq \mathrm{k} \leq \mathrm{N}\}$ are the RPmin of the $\mathrm{N}$ previous spinning period.

- $\mathrm{dRmax}=\mathrm{R} \max -\mathrm{R} \min , \mathrm{dRP} \max =\mathrm{RPmax}-\mathrm{RPmin}$

\subsection{First Method: Ratio Pulses Factor RP}

Firstly, the Ratio Pulse factor RP (RPmax, RPmin, dRP) for a flare with the following parameters are gotten:

- $\quad$ (Tlanuch $=0.2 \mathrm{~s}, \quad$ Trise $=0.5 \mathrm{~s}, \quad \mathrm{Tf}=3.5 \mathrm{~s}, \quad \mathrm{~Tb}=4$, Tmax $=2000 \mathrm{k}$, Tmin $=850 \mathrm{k}$ ).

- $\quad$ speed $=\{500,1000,1500,2000,2500\} \mathrm{pixel} / \mathrm{s}$.

- $\operatorname{Tr}=\{0.01,0.05,0.1,0.2,0.25,0.3\}$.

Taking into account that the maximum temperature of usual targets is less than $\underline{\mathbf{T}}_{\mathrm{th} 1}=(\mathbf{1 1 0 0 - 1 1 5 0 ) \mathbf { k }}$, a threshold can be defined to detect the appearance of the flare $R_{t h 1}=2$. All the data are stated and resumed in the tables (5-9) and two cases are drawn in Fig 12

From these tables:

- It is clear that there are two cases of detection:

- Detection before the pulses separation (first phase): the condition of that is the flare reaches, in this phase, a permanent temperature which is a temperature more than the (Tth $1=1150 \mathrm{k})$ with different amounts (in some case it reaches 1500k) .

- Detection after the pulses separation (second phase): if the separation takes place before reaching the permanent temperature, the condition becomes reaching the threshold temperature Tth 1 .

- From table 6 RPmax is not capable to be a parameter for detecting the flare. the raison of that, if it could not detect the flare in the first phase it will lose it as in the second phase its value will be the target own which is high one.

- From table $7 \mathbf{d R P}$ is independent of the temperature profile and depends mainly on the movement profile.

- From tables (7-9) dRP cannot detect the flare in the first phase. the raison for that is before this instant, the instant of separation, the pulses of the target and the flare are superposed, so all the pulses have the same correlation characteristics which means that $(\mathrm{RPmin} \approx \mathrm{RPmax}, \mathrm{dRP}$ $\approx 0$ ). On the contract after the separation two groups of pluses appear each has its own correlation characteristics related to its own temperature profile.

- From (6,8 and 9) RPmin can detect the flare effectively in the second phase as it expresses the flare ratio factor 
independently from the target one which means that the flare will be detected when its temperature reaches the threshold one Tth1. On the contrast in the first phase, it cannot detect the flare unless its temperature reaches a permanent value which is some time high one relatively to the threshold one.

- In Fig 12(b) the flare temperature increasing is so slow in the first phase that the pulse peak expresses the target one which means ( $\mathrm{RPmax} \approx \mathrm{RPmin} \approx \mathrm{RPtarget})$. And as a result the RPmin cannot detect in this case in the first phase and it has to wait for the second phase starting.

Table 5. The required time for detection (Treq) and the temperature at the detection (Tdet) using $(\mathrm{RPmin}<2)$

condition for several $\mathrm{Tr}$ and speed=1000 pixel/s.

\begin{tabular}{|c|c|c|c|c|c|c|}
\hline TR & $\mathbf{0 . 0 1}$ & $\mathbf{0 . 0 5}$ & $\mathbf{0 . 1 0}$ & $\mathbf{0 . 2 0}$ & $\mathbf{0 . 2 5}$ & $\mathbf{0 . 3}$ \\
\hline Treq(s) & 0.01 & 0.04 & 0.06 & 0.12 & 0.12 & 0.12 \\
\hline Tdet(k) & 1575 & 1480 & 1370 & 1370 & 1288 & 1230 \\
\hline
\end{tabular}

Table 6. The required time for detection (Treq) and the temperature at the detection (Tdet) using $(\mathrm{RPmax}<2)$

condition for several $\operatorname{Tr}$ and speed $=1000 \mathrm{pixel} / \mathrm{s}$.

\begin{tabular}{|c|c|c|c|c|c|c|}
\hline TR & $\mathbf{0 . 0 1}$ & $\mathbf{0 . 0 5}$ & $\mathbf{0 . 1 0}$ & $\mathbf{0 . 2 0}$ & $\mathbf{0 . 2 5}$ & $\mathbf{0 . 3}$ \\
\hline Treq(s) & $0.02 \mathrm{~s}$ & $0.05 \mathrm{~s}$ & $0.07 \mathrm{~s}$ & none & none & none \\
\hline Tdet(k) & 1840 & 1575 & 1425 & & & \\
\hline
\end{tabular}

Table 7. The required time for detection (Treq) and the temperature at the detection (Tdet) using (Drp>2) condition for several $\mathrm{Tr}$ and speed $=1000 \mathrm{pixel} / \mathrm{s}$.

\begin{tabular}{|c|c|c|c|c|c|c|}
\hline TR & $\mathbf{0 . 0 1}$ & $\mathbf{0 . 0 5}$ & $\mathbf{0 . 1 0}$ & $\mathbf{0 . 2 0}$ & $\mathbf{0 . 2 5}$ & $\mathbf{0 . 3}$ \\
\hline Treq(s) & 0.13 & 0.13 & 0.13 & 0.13 & 0.13 & 0.13 \\
\hline Tdet(k) & 2000 & 1900 & 1680 & 1400 & 1317 & 1250 \\
\hline
\end{tabular}

Table 8. the required time for detection (Treq(s) ) using the conditions (Drp $>2, \operatorname{Rpmin}<2, \operatorname{Rpmax}<2$ ) for several speeds

\begin{tabular}{|c|c|c|c|c|}
\hline apeed (pixel/s) & $\mathbf{5 0 0}$ & $\mathbf{1 0 0 0}$ & $\mathbf{1 5 0 0}$ & $\mathbf{2 0 0 0}$ \\
\hline RPmin $<\mathbf{2}$ & 0.16 & 0.12 & 0.08 & 0.06 \\
\hline RPmax $<2$ & 0.17 & none & none & none \\
\hline dRPmin $>\mathbf{2}$ & 0.24 & 0.13 & 0.09 & 0.07 \\
\hline
\end{tabular}

Table 9. The required time for detection (Treq(s) ) using the conditions (Drp $>2, R p m i n<2$, Rpmax $<2$ ) for several speeds

\begin{tabular}{|c|c|c|c|c|}
\hline Speed (pixel/s) & $\mathbf{5 0 0}$ & $\mathbf{1 0 0 0}$ & $\mathbf{1 5 0 0}$ & $\mathbf{2 0 0 0}$ \\
\hline RPmin $<\mathbf{2}$ & 0.08 & 0.06 & 0.06 & 0.06 \\
\hline RPmax $<2$ & 0.08 & 0.07 & 0.07 & none \\
\hline dRPmin $>\mathbf{2}$ & 0.24 & 0.13 & 0.09 & 0.07 \\
\hline
\end{tabular}

\subsection{Second Method: Instant Ratio Factor $R$}

By the same manner, the Ratio Factor R (Rmax, Rmin, dR) for a flare with the following parameters are gotten:

- $\quad$ (Tlanuch $=0.2 \mathrm{~s}, \quad$ Trise $=0.5 \mathrm{~s}, \quad \mathrm{Tf}=3.5 \mathrm{~s}, \quad \mathrm{~Tb}=4$, $\mathrm{Tmax}=2000 \mathrm{k}, \mathrm{Tmin}=850 \mathrm{k}$ )

- $\quad$ speed $=\{500,1000,1500,2000,2500\}$ pixel $/ \mathrm{s}$.

- $\operatorname{Tr}=\{0.01,0.05,0.1,0.2,0.25,0.3\}$.

Taking into account that the maximum temperature of usual targets is less than $\underline{\mathbf{T}}_{\mathbf{t h 1}}=\mathbf{( 1 1 0 0 - 1 1 5 0 ) \mathbf { k }}$, a threshold can be defined to detect the appearance of the flare $R_{t h 1}=2$. All the data are stated and resumed in the tables (10-11).

From these tables :

- It is clear that there are have not two phases:

- Detection before the pulses separation (first phase)

- Detection after the pulses separation (second phase)

- $\quad \underline{\mathbf{R} m a x}$ is not capable to be a parameter for detecting the flare. The raison of that it expresses the ratio of the target ownin lower temperature which is in this case the real target.

- Clearly, (dR, Rmin) are independent of the movement profile and depends mainly on the temperature profile.

- $\quad$ Rmin can detect the flare effectively in the both phases. The raison of that it expresses the ratio of the target owning the higher temperature which is in this case the flare, so without needing to reach a permanent temperature higher than the threshold one. It is clear that the detection will happen at this threshold independently of any other parameter.

- $\quad \underline{\mathbf{R}}$ can detect the flare in both the phases .

The flowchart of using the condition ( $\mathbf{R m i n}<2$ ) or $(\mathbf{d R}>\mathbf{2 . 5})$ in the CAT seeker is stated in Fig 13.

TABLE 10. THE REQUIRED TIME FOR DETECTION (TREQ) AND THE TEMPERATURE AT THE DETECTION (TDET) USING $($ RMIN $<2)$ CONDITION FOR SEVERAL TR AND SPEED=1000 PIXEL/S.

\begin{tabular}{|c|c|c|c|c|c|c|}
\hline TR & $\mathbf{0 . 0 1}$ & $\mathbf{0 . 0 5}$ & $\mathbf{0 . 1 0}$ & $\mathbf{0 . 2 0}$ & $\mathbf{0 . 2 5}$ & $\mathbf{0 . 3}$ \\
\hline Treq(s) & 0.01 & 0.02 & 0.04 & 0.07 & 0.08 & 0.10 \\
\hline Tdet(k) & 1575 & 1230 & 1200 & 1200 & 1200 & 1180 \\
\hline
\end{tabular}

Table 11. the required time for detection (Treq) and the temperature at the detection (Tdet) using (DR>2.5) condition for several $\mathrm{Tr}$ and speed $=1000 \mathrm{pixel} / \mathrm{s}$.

\begin{tabular}{|c|c|c|c|c|c|c|}
\hline TR & $\mathbf{0 . 0 1}$ & $\mathbf{0 . 0 5}$ & $\mathbf{0 . 1 0}$ & $\mathbf{0 . 2 0}$ & $\mathbf{0 . 2 5}$ & $\mathbf{0 . 3}$ \\
\hline Treq(s) & 0.01 & 0.02 & 0.04 & 0.07 & 0.08 & 0.10 \\
\hline Tdet(k) & 1575 & 1230 & 1200 & 1200 & 1200 & 1180 \\
\hline
\end{tabular}

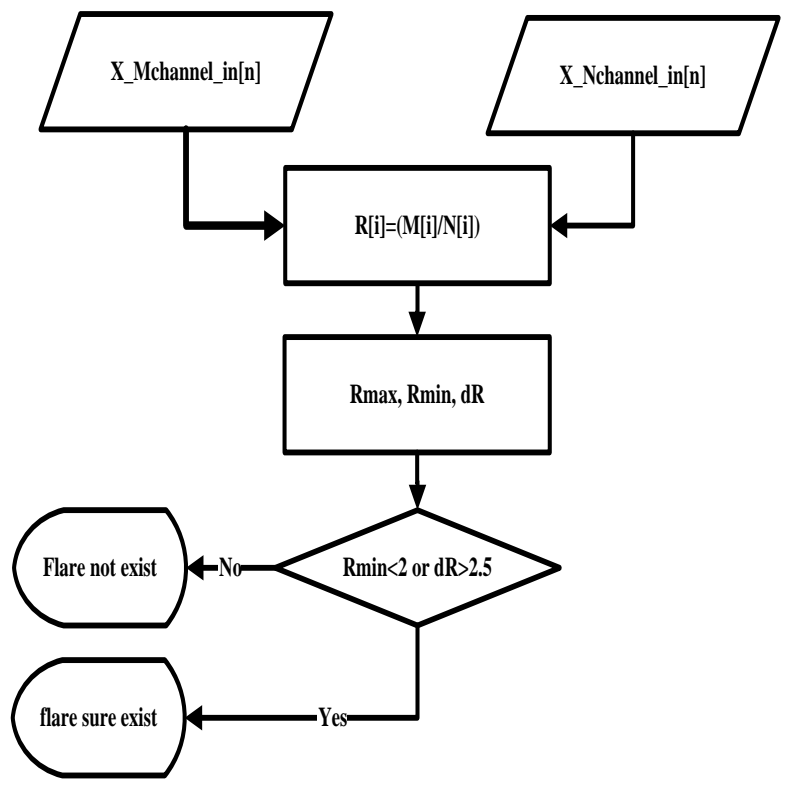

Fig 13: The flowchart of the flare detection using the Ratio Pulsese Factor (R). 
Table 12. Comparison of the flare detection methods

\begin{tabular}{|c|c|c|c|c|}
\hline $\begin{array}{c}\text { Detecting } \\
\text { parameter }\end{array}$ & $\begin{array}{c}\text { First } \\
\text { phase }\end{array}$ & $\begin{array}{c}\text { Second } \\
\text { phase }\end{array}$ & $\begin{array}{c}\text { Using } \\
\text { movement } \\
\text { effect }\end{array}$ & $\begin{array}{c}\text { Using } \\
\text { temperatu } \\
\text { re profile }\end{array}$ \\
\hline PW & yes & no & yes & no \\
\hline PP & no & yes & yes & no \\
\hline ARM & limited & yes & little & yes \\
\hline ARS & limited & yes & little & yes \\
\hline RPmax & limited & no & yes & yes \\
\hline RPmin & limited & yes & yes & yes \\
\hline dRP & no & yes & yes & no \\
\hline Rmax & no & no & no & no \\
\hline Rmin or dR & yes & yes & no & yes \\
\hline
\end{tabular}

Table 13. Comparison of the effective flare detection methods

\begin{tabular}{|c|c|c|c|c|}
\hline $\begin{array}{c}\text { Detecting } \\
\text { parameter }\end{array}$ & $\begin{array}{c}\text { First } \\
\text { phase }\end{array}$ & $\begin{array}{c}\text { Second } \\
\text { phase }\end{array}$ & $\begin{array}{c}\text { Using } \\
\text { movement } \\
\text { effect }\end{array}$ & $\begin{array}{c}\text { Using } \\
\text { temperatu } \\
\text { re profile }\end{array}$ \\
\hline ARM & limited & yes & little & yes \\
\hline Rmin & yes & yes & no & yes \\
\hline
\end{tabular}

\section{COMPARISON OF THE METHODS.}

The more effective and robust method for detecting the flare existence of appearance is the method which can exploit the movement and the temperature of the flare to detect it in both the first phase and the second one one. The first phase is the duration between the flare launch and the instant when the pulses of the flare and those of the target are separated in the information signal, and the second is the phase after this separation. Some methods, like (PW, RPmax, Rmax) as shown in table 12, cannot detect in the second phase which means the flare detection can be missed and the existence detection is not possible. Other methods, like (PP, ARM, ARS, RPmax, RPmin, dRP, Rmax), cannot detect in the first phase, totally or limitedly, which means losing the detection or takes long time for detection.

The methods which can detect in both the phases, as shown in table 13, are (AR, Rmin). AR depends mainly on the temperature profile and the main channel amplitude profile which makes it under the possible influence of the fluctuations or the perturbations on the main channel signals resulting from a possible unstable navigation or a strong background. Also, Rmin cannot take into account the target with high temperature.

\section{CONCLUSION}

Designing and implementing effective IRCCM needs good simulation tools and good understanding of the signals generated from the seeker as a result of the existence of a target in the FOV. And one of the important steps is the flare detection. In this work, the flare is modeled by modeling its movement in the FOV and modeling its temperature profile. This model takes 6 variable inputs for the flare temperature and 2 for its positions; a as result a large scanning of the possible flare scenarios could be covered. Several methods are stated, some depend on the main channel amplitude or the secondary channel amplitude like (ARM, ARS). Others used the two color characteristics of the seeker like (RPmax, $\mathrm{RPmin}, \mathrm{dRP}$ ) group and (Rmax, Rmin, $\mathrm{dR}$ ) group.

\section{REFERENCES}

[1] G. Gerson and A. K. Rue, "Tracking Systems," Chap. 22 in The Infrared Handbook, G. J. Zissis and W. L. Wolfe, Eds., RIM, Ann Arbor, MI(1985).
[2] K. Seyrafi and S. A. Hovanessian, Chap. 7 in Introduction to Electooptical Imaging and Tracking Systems, pp. 193-220, Artech House,Norwood, MA(1993).

[3] R. Legault, "Reticle and Image Analyses," Chap. 17 in The Infrared Handbook, revised ed., W. L. Wolfe and G. J. Zissis, Eds., pp. 17-1-17-49, Environmental Research Institute of Michigan, Ann Arbor, MI(1985).

[4] J. S. Accetta and D. L. Shymaker, Eds., "The Infrared \& Electro-Optical Systems Handbook", SPIE Press (1993).

[5] J. S. Oh, K. S. Doo, S. G. Jahng, and J. S. Choi, "A New Counter-Countermeasure Algorithm for Two-Color Infrared Seekers," Optical Engineering, vol. 40, No. 8 (2001).

[6] S Y AlchekhYasin, A R Yrfanean, M R Mosavi and A Mohammadi, "Modeling and Simulation of the Active Jammer Effect in the Crossed Array Detectors Infrared Seeker," International Journal of Computer Applications 72(4):15-22, June 2013.

[7] A R Yrfanean, M R Mosavi, A Mohammadi and Alchekh S Y Yasin, "Improving the Target Position Detection in the Crossed Array Detectors Seeker by Categorizing the FOV up to the Pulses Distribution," International Journal of Computer Applications72(4):28-36, June 2013.

[8] Z. W. Chao and J. L. Chu, "General Analysis of Frequency-Modulation Reticles," Opt. Eng. 27, 440442 (1988)

[9] M. A. Porras, J. Alda, and E. Bernabeu, "AmplitudeModulated and Frequency-Modulated Reticle Responses of Gaussian Beams," Optical Engineering, Vol. 30, No. 12 (1991)

[10] R. G. Driggers, C. E. Halford, and G. D. Boreman, "Use of Spatial Light Modulators in Frequency Modulation Reticle Trackers," Optical Engineering. Vol. 29, No. 11, pp. 1398-1403 (1990).

[11] W. Haifeng, L. Zhi, Z, Qing, and S. Xinzhi, "A Double Infrared Image Processing System Using Rosette Scanning," Proceeding. SPIE, Vol. 2894, pp. 2-10 (1996).

[12] S. G. Jahng, H. K. Hong, S. H. Han, and J. S. Choi, "Dynamic Simulation of The Rosette Scanning Infrared Seeker and an IRCCM using The Moment Technique," Opt. Eng. 38(5), 921-928 (1999).

[13] J. S. Oh, K. S. Doo, S. G. Jahng, D. S. Seo, and J. S. Choi, "Novel Adaptive Digital Signal Processing Algorithm for a Stationary Reticle Seeker," Optical Engineering, Vol. 39, No. 1 0, pp. 2797-2803, 2000.

[14] "Development of an IR Signature Model," [Online]. available:https://dspace.lib.cranfield.ac.uk/bitstream/.../C HAPTER_6_july26.pdf.

[15] M. R. Mosavi, M. Asadpour, and R. Kalili,“"Comparing Performance of Two Infrared Anti-Jamming Methods using Fuzzy System and Neural Network," 2007 Congress on Intelligent and Fuzzy Systems, Ferdowsi University of Mashhad, Iran (2007).

[16] M. R. Mosavi, M. Asadpour, and H. A. Amerim,"Design and Simulation of an Infrared Jammer Source for an Infrared Seeker," IEEE Conference on Signal Processing, Communications, and Networking, India (2008) 